\title{
Treatment of posterior cruciate ligament avulsion fractures of the tibia using a toothed plate and hollow lag screw
}

Wei $\underline{\text { Chen }}^{1}$, Bs, Wei $\underline{L u O}^{1}$, Ms, Zhiqing $\underline{\text { Chen }}^{1}$, BS, Yi $\underline{\text { Jiang }}^{1}$, BS

INTRODUCTION To investigate the feasibility and clinical efficacy of using a toothed plate and hollow lag screw in the surgical treatment of posterior cruciate ligament $(P C L)$ avulsion fractures of the tibia.

METHODS A total of 21 patients were treated with open reduction and internal fixation using a toothed plate and hollow lag screw, through a posteromedial approach using an inverted L-shaped incision. The patients were allowed appropriate functional exercises, including knee flexion and extension, after removal of the plaster at postoperative weeks 3-6. The follow-up period was between six months and two years.

RESULTS This was a retrospective study of patients with PCL avulsion fractures of the tibia caused by road traffic accidents $(n=9)$, sports-related injuries $(n=6)$, falls $(n=5)$ and machinery-related injuries $(n=1) .20$ patients presented with fresh fractures and one with an old fracture. The patients (13 men, eight women) had a mean age of 41.5 (range 19-72) years. Anatomical reduction of the fracture and satisfactory fixation were achieved in all 21 patients. Bony union was achieved in all patients at 8-12 weeks after surgery. Six months after surgery, knee flexion was $121.9^{\circ} \pm 10.4^{\circ}$ and extension was $0^{\circ}$. According to the Lysholm Knee Scoring Scale, 19 patients were rated as 'excellent' and two as 'good'. CONCLUSION The use of a toothed plate and hollow lag screw could be a simple and reliable approach for PCL avulsion fractures of the tibia. Patients achieved good knee function after surgery.

Keywords: fracture, internal fixation, posterior cruciate ligament, toothed plate

\section{INTRODUCTION}

The posterior cruciate ligament $(\mathrm{PCL})$ is an important structure that helps to maintain the stability of the knee during flexion and rotation. PCL injuries may lead to instability that allows the tibia to move in the posterior direction, impeding the ability to keep the back of the knee joint straight and inducing degenerative changes in the knee in the long term. PCL injuries are less common than anterior cruciate ligament (ACL) injuries. ${ }^{(1)}$ Since the $\mathrm{PCL}$ is strong, avulsion fractures of its attachment are common. ${ }^{(2)}$ Currently, the most common treatment used for complete restoration of PCL function after an avulsion fracture is surgery using internal fixation.

Surgical treatments for PCL avulsion fractures of the tibia include arthroscopic repair as well as open reduction and internal fixation (ORIF). Although arthroscopic repair is minimally invasive, the technique has several disadvantages, including the fact that such fixation and reduction are more challenging to perform than open surgery, the long learning curve and a requirement for specialised equipment, possibly making arthroscopic repair difficult to implement in primary-level hospitals. For these reasons, most hospitals treat PCL avulsion fractures of the tibia with ORIF in the posterior knee.

There is currently a wide variety of materials available for use in internal fixation, including hollow lag screws, steel wires, absorbable screws, suture anchors and straddle nails. ${ }^{(3,4)}$ The selection of material is limited by the nature of the fracture. Furthermore, problems can arise from the use of these materials.
These include unstable fixation when pressure is applied on the fracture to bring the fragments together and the preclusion of early postoperative functional exercise.

Therefore, our aim was to identify a suitable method for ORIF that could be used to treat a variety of fractures, while ensuring stable fixation and allowing early postoperative functional exercise. We retrospectively analysed 21 patients with PCL avulsion fractures of the tibia who were treated in our hospital between 2009 and 2012 with ORIF in the posteromedial knee, using a toothed plate (also named spiked washer by some companies) and hollow lag screw. The objective of this study was to evaluate the therapeutic effect of ORIF using a toothed plate and hollow lag screw, in order to determine whether this simple internal fixation method could produce satisfactory treatment outcomes.

\section{METHODS}

This was a retrospective study of 21 consecutive patients with PCL avulsion fractures of the tibia who were treated in our hospital between March 2009 and August 2012. All patients were treated with ORIF using a toothed plate and hollow lag screw. The decision to perform surgery was based on the presence of a fresh fracture displacement $>10 \mathrm{~mm}$ or failure of conservative treatment of an old fracture with obvious knee instability. The exclusion criteria were contraindication to surgery, serious complications that precluded surgical intervention, non-displaced fracture fragments or fragment displacement $<10 \mathrm{~mm}$. 
The main symptoms and signs included knee pain, joint swelling, limited mobility and a positive drawer test (carried out under anaesthesia, before surgery). Routine preoperative knee radiography, computed tomography $(\mathrm{CT})$ and magnetic resonance (MR) imaging were used to confirm the diagnosis of PCL avulsion fractures of the tibia. The avulsion fracture was diagnosed based on the following criteria: a clear history of trauma; knee pain and swelling after injury; positive posterior drawer test; PCL avulsion fractures of the tibia shown by radiography and $\mathrm{CT}$; and an intact PCL on MR imaging. Preoperative and postoperative knee function was evaluated using the Lysholm Knee Scoring Scale. ${ }^{(5)}$ The study was approved by the Ethics Committee of Tianjin Third Central Hospital, Tianjin, China, and all participants provided written informed consent.

Epidural anaesthesia was used for surgery. Surgery was carried out with the patient in the prone position, with a pneumatic tourniquet used to minimise bleeding. A $12 \mathrm{~cm}$ (approximate) inverted L-shaped incision was made in the skin and subcutaneous tissue of the posterior aspect of the knee, along the transverse crease of the popliteal fossa, inwards to the edge of the medial head of the gastrocnemius muscle and then distally along the medial aspect of the lower leg. After identification and protection of the small saphenous vein and medial sural cutaneous nerve, the deep fascia was incised medial to these structures to expose the medial head of the gastrocnemius and semitendinosus. The medial head of the gastrocnemius muscle and the popliteal vessels and nerves were retracted laterally to expose the posterior joint capsule, and the joint capsule was incised longitudinally to expose the PCL and avulsion fracture fragments. Any blood clots or soft tissue embedded in the fracture fragments were removed. In patients with an old fracture, any granulation tissue on the fracture fragments was completely removed to expose a fresh mattress of bone on the intercondylar eminence of the tibia. Then, with a thick cushion placed in front of the patient's thigh, the anterior drawer test was applied to the lower leg and the injured knee was bent to obtain anatomic reduction of the fracture fragments. Two Kirschner wires were used for temporary fixation. When the appropriate position was accurately identified by $\mathrm{C}$-arm fluoroscopic examination, a toothed plate (Fig. 1; Johnson \& Johnson, New Brunswick, NJ, USA) of the appropriate diameter (selected based on the fracture fragment size) was placed on the fracture fragments and hammered to embed the teeth around the plate into the PCL. A guide needle was drilled vertically into the plate, and a hollow lag screw of the appropriate diameter was screwed and fixed, with pressure applied to bring the fragments together (Fig. 2). For large fracture fragments, an additional cancellous lag screw or absorbable screw was needed for fixation. The Kirschner wires and guide needle were then removed, and the posterior drawer test was performed; if the test was negative, the wound was closed with sutures.

The knee was fixed at $30^{\circ}$ flexion using a conventional plaster cast and quadriceps exercises were started from the first day after surgery. If the fracture fragment was large and internal fixation was considered stable, the plaster was removed after three

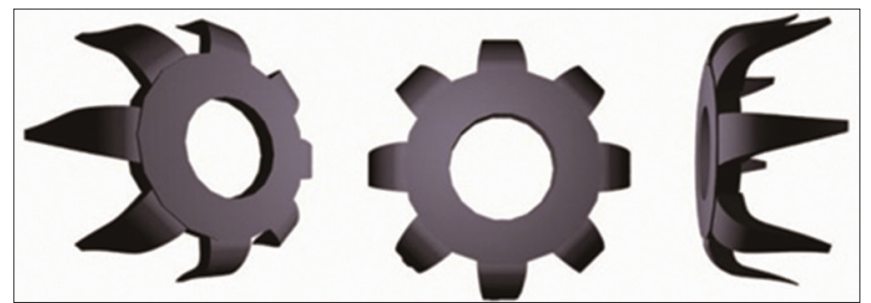

Fig. 1 Schematic diagram of the toothed plate.

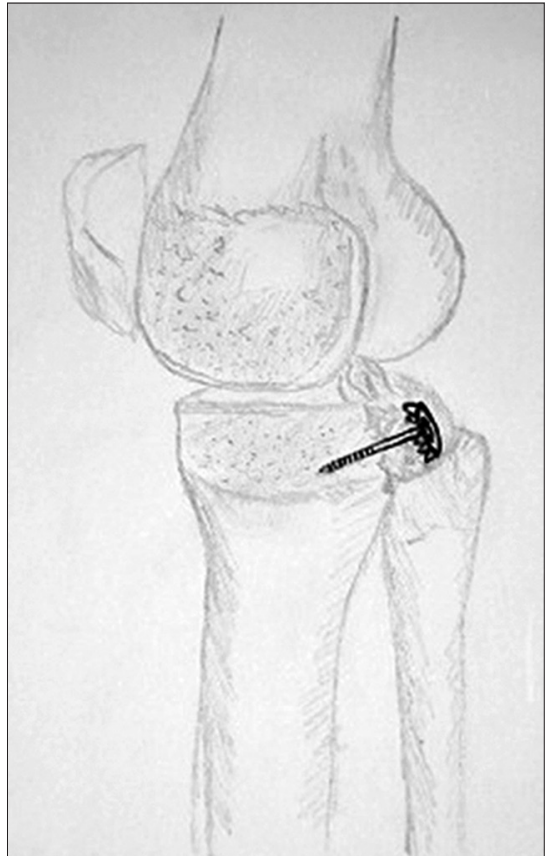

Fig. 2 Schematic diagram shows the fixation of a posterior cruciate ligament avulsion fracture with a toothed plate.

weeks, allowing active and passive knee functional exercises to be performed. However, if the fracture fragment was small or crushed, plaster removal, and active and passive knee functional exercises were delayed until six weeks after surgery. Patients were allowed to walk up and down stairs with the aid of crutches, with partial weight bearing on the injured limb, only six weeks after surgery. After three months, the patients were allowed to exercise without the aid of crutches, with full weight loading on the injured limb.

Outpatient follow-up was conducted at six weeks, eight weeks, three months, six months, one year and two years after surgery. The outcome measures included the duration of surgery, healing time, knee stability, range of knee motion and complications (such as infection, delayed union or non-union, and translocation or fracture of the plate and screw). In addition, knee function was assessed according to the Lysholm Knee Scoring system, ${ }^{(5)}$ which includes eight items: pain (0-25 points); swelling (0-10 points); limp ( $0-5$ points); support using cane or crutches $(0-5$ points); climbing stairs ( $0-10$ points); instability ( $0-25$ points); squatting (0-5 points); and locking sensation in the knee (0-15 points). Treatment efficacy was classified as follows, based on the Lysholm Knee Scoring Scale: 'excellent' for > 85 points; 'good' for 70-85 points; 'intermediate' for 60-69 points; and 'poor' for $<60$ points. 
Data analysis was performed using SPSS version 13.0 (SPSS Inc, Chicago, IL, USA). Data was presented as mean \pm standard deviation. Student's paired $t$-tests were used for intergroup comparison of Lysholm knee scores before and after surgery. A p-value $<0.05$ was considered statistically significant.

\section{RESULTS}

The patients (13 men, eight women) had a mean age of 41.5 (range 19-72) years. The causes of injury were road traffic accidents $(n=9)$, falls $(n=5)$, sports-related $(n=6)$ and machinery-related $(n=1) .20$ patients presented with fresh fractures (defined as having occurred within the last two months), while only one presented with an old fracture. 15 patients presented with $\mathrm{PCL}$ avulsion fractures of the tibia only; additionally, of the remaining six patients, three also had a lateral meniscus injury, one had a medial collateral ligament injury, one had an ipsilateral patellar fracture and one had an ipsilateral patellar tendon rupture combined with an open knee joint injury. CT identified simple fractures in 11 patients and comminuted fractures in ten patients. MR imaging showed that the cruciate ligament behind the sagittal plane was twisted and shrunken, but that the connection between the avulsed fragments and the PCL was not broken. T2-weighted images showed high signal strength of the fracture line at the posterior of the plateau.

The operation was uneventful in all 21 patients, with a mean duration of $65.5 \pm 13.7$ (range 40-95) minutes. Intraoperative blood loss was $54.3 \pm 15.0$ (range 30-80) mL. All the patients were followed up for an average of 14 (range 6-24) months. Successful anatomical reduction of the fracture fragments, satisfactory fixation and good wound healing were achieved in all the patients. The average fracture healing time was $10.2 \pm 1.7$ (range 8-12) weeks. At the last follow-up, the range of knee motion was assessed to be $121.9^{\circ} \pm 10.4^{\circ}$ for flexion and $0^{\circ}$ for extension. No posterior sag was found in 14 patients, while grade 1 and 2 sag was found in four and three patients, respectively; no patient had grade 3 sag. Six months after surgery, the Lysholm knee score among all the patients averaged $93.6 \pm 5.9$ (range 76-99) points. Treatment efficacy was determined as 'excellent' in 19 patients and 'good' in two patients. Furthermore, the postoperative Lysholm knee scores improved compared with the pre-surgery scores (Tables I and II). None of the 21 patients were found to have complications, such as postoperative wound infection, fracture displacement, plate and screw breakage, non-union and delayed healing.

To illustrate the aforementioned findings, we include a brief description of Case 1, a 25-year-old man with a PCL avulsion fracture of the tibia combined with patellar ligament rupture of the right knee. Preoperative CT demonstrated a PCL avulsion fracture of the tibia of the right knee, with obvious displaced fracture fragments (Fig. 3a). Preoperative MR imaging showed an intact PCL and avulsion fracture of the tibia of the right knee (Fig. 3b). Three months after surgery, radiography revealed that the fracture had healed well (Figs. 3c \& d). After six months, clinical examination demonstrated good joint function in the patient (Figs. 3e \& f).
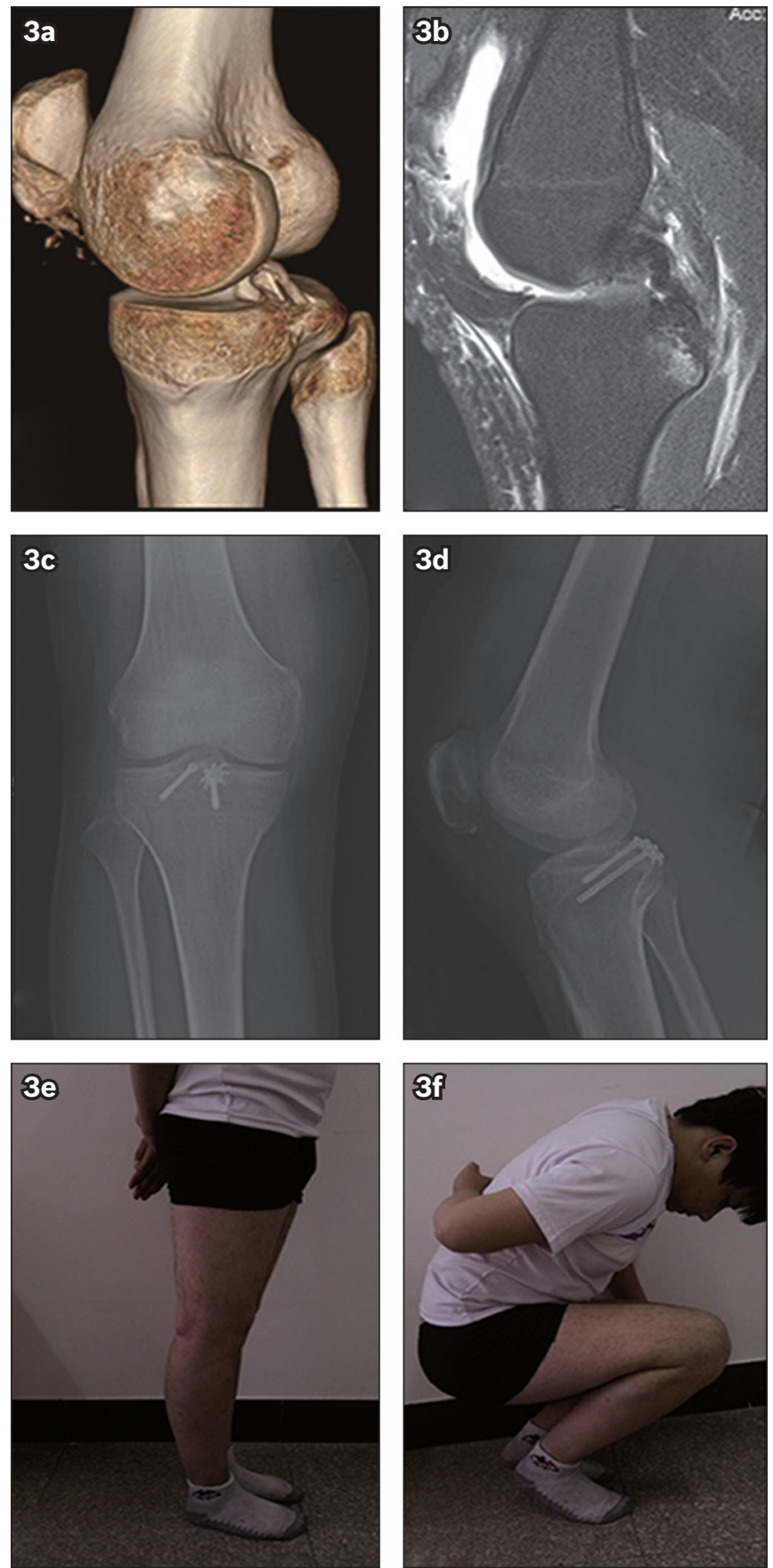

Fig. 3 A 25-year-old man with a posterior cruciate ligament (PCL) avulsion fracture and patellar ligament rupture of the right knee. (a) CT image of the right knee shows a PCL avulsion fracture and fragment displacement. (b) MR image shows a complete PCL and an avulsed fracture of the tibia. (c \& d) Radiographs show bone union three months after surgery. (e \& f) Photographs show the patient with good right knee function six months after surgery.

\section{DISCUSSION}

The main finding of the present study was that surgical management of PCL avulsion fractures of the tibia using a toothed plate and hollow lag screw was associated with a low incidence of non-union and knee instability, as well as significant improvements in the Lysholm knee score compared with the presurgery score. The early efficacy and full restoration of normal daily activities in all patients suggests that this treatment method may achieve good results and, hence, merits further investigation. 
Table I. Characteristics and clinical data of the patients $(n=21)$.

\begin{tabular}{|c|c|c|c|c|c|c|c|c|}
\hline $\begin{array}{l}\text { Gender/ } \\
\text { age (yr) }\end{array}$ & Cause of injury & $\begin{array}{l}\text { Duration } \\
\text { of surgery } \\
\text { (min) }\end{array}$ & $\begin{array}{c}\text { Quantity } \\
\text { of bleeding } \\
\text { (mL) }\end{array}$ & $\begin{array}{c}\text { Follow-up } \\
\text { time } \\
\text { (mth) }\end{array}$ & $\begin{array}{c}\text { Fracture } \\
\text { healing } \\
\text { time (wk) }\end{array}$ & $\begin{array}{c}\text { Range } \\
\text { of knee } \\
\text { motion }\left(^{\circ}\right)\end{array}$ & $\begin{array}{l}\text { Preoperative } \\
\text { Lysholm } \\
\text { score }\end{array}$ & $\begin{array}{l}\text { Postoperative } \\
\text { Lysholm } \\
\text { score }\end{array}$ \\
\hline Male/19 & Road traffic accident & 40 & 30 & 6 & 8 & 100 & 4 & 99 \\
\hline Male/26 & Sports-related & 45 & 40 & 12 & 10 & 110 & 19 & 96 \\
\hline Female/24 & Sports-related & 50 & 50 & 24 & 12 & 120 & 43 & 95 \\
\hline Male/31 & Sports-related & 56 & 60 & 24 & 12 & 130 & 38 & 95 \\
\hline Female/50 & Fall & 60 & 70 & 6 & 8 & 140 & 34 & 95 \\
\hline Female/36 & Machinery-related & 60 & 50 & 12 & 10 & 120 & 48 & 87 \\
\hline Female/39 & Road traffic accident & 65 & 50 & 18 & 12 & 120 & 10 & 81 \\
\hline Male/53 & Fall & 68 & 40 & 18 & 12 & 110 & 2 & 76 \\
\hline Male/51 & Road traffic accident & 70 & 80 & 6 & 8 & 135 & 43 & 96 \\
\hline Male/40 & Road traffic accident & 75 & 80 & 12 & 10 & 140 & 48 & 95 \\
\hline Female/52 & Road traffic accident & 75 & 50 & 6 & 8 & 120 & 19 & 96 \\
\hline Female/54 & Fall & 80 & 60 & 18 & 10 & 120 & 16 & 96 \\
\hline Male/28 & Sports-related & 90 & 50 & 24 & 10 & 130 & 36 & 99 \\
\hline Female/42 & Road traffic accident & 95 & 70 & 18 & 12 & 120 & 31 & 99 \\
\hline Male/38 & Road traffic accident & 60 & 50 & 12 & 12 & 125 & 10 & 99 \\
\hline Female/36 & Road traffic accident & 65 & 30 & 6 & 8 & 130 & 19 & 95 \\
\hline Male/42 & Road traffic accident & 62 & 40 & 12 & 8 & 120 & 10 & 96 \\
\hline Male/36 & Sports-related & 70 & 50 & 18 & 10 & 110 & 36 & 96 \\
\hline Male/58 & Fall & 65 & 60 & 18 & 12 & 120 & 19 & 95 \\
\hline Male/72 & Fall & 50 & 50 & 12 & 10 & 110 & 34 & 90 \\
\hline
\end{tabular}

Table II. Lysholm knee scores before and after surgery.

\begin{tabular}{lcc}
\hline Time & No. of patients & Lysholm score* \\
\hline Before surgery & 21 & $25.3 \pm 14.7$ \\
After surgery & 21 & $93.6 \pm 5.9$ \\
p-value & - & $<0.001$ \\
\hline
\end{tabular}

* Data presented as mean \pm standard deviation.

As the PCL is a strong ligament, a force that damages it often leads to related injuries in the tibia or femur. ${ }^{(6)}$ Out of the 21 cases in the present study, road traffic accidents (42.9\%) were responsible for the largest number of avulsion fractures, followed by sports-related injuries (28.6\%) and falls (23.8\%). Our findings are thus in agreement with those of previous studies. ${ }^{(7,8}$ For example, Schulz et al reported that motor vehicle accidents and athletic injuries accounted for $45 \%$ and $40 \%$ of their cases, respectively. ${ }^{(8)}$ The most common mechanism underlying PCL avulsion fractures of the tibia in road traffic accidents is dashboard collision, in which a direct force is applied to the proximal part of the tibia in an anterior-to-posterior direction, with the knee in flexion. ${ }^{(7,8)}$ Another mechanism is associated with a proximal tibial fracture caused by a force on the lateral joint capsule and the ligaments of the tibial meniscus, ${ }^{(9)}$ which is often combined with a lateral tibial avulsion fracture (Segond fracture).

Although the relative merits of conservative and surgical managements are controversial, surgery is known to be superior in young and active individuals who require good lower limb mobility and function. Conservative treatment may increase the risk of articular cartilage degeneration (which in turn leads to arthritis), meniscal tears and quadriceps atrophy. ${ }^{(10,11)} \mathrm{PCL}$ repair in the acute phase can effectively prevent these changes. ${ }^{(11)}$ Since the abnormal biomechanics of a knee without the PCL can enhance the risk of knee degeneration, the present study observed cases in which an interventional approach was used to treat PCL avulsion fractures of the tibia in order to restore normal knee kinematics and prevent knee degeneration. There is evidence to suggest that surgical ORIF should be actively implemented when fracture fragment displacement is more than $1 \mathrm{~cm}$ or when conservative treatment has failed. ${ }^{(12,13)}$

The current surgical options for PCL avulsion fractures of the tibia include arthroscopic repair and ORIF. First reported in 1995, arthroscopic treatment of tibial avulsion fractures has since received much attention with the progress of arthroscopic techniques. ${ }^{(14-17)}$ Although arthroscopic repair is minimally invasive and can simultaneously treat related injuries such as meniscal and synovial damage, it is a relatively complicated procedure with high technical requirements and a long learning curve, and requires specific equipment. In addition, reduction and fixation are more challenging with arthroscopic techniques than with open surgery. The application of the open posteromedial approach, which avoids nerves and blood vessels, has the potential to provide a clearer view of the broken ends of the fractured bone, and allows for complete fracture reduction and successful fixation of the bone fracture fragments. In 2007, Sasaki et $\mathrm{al}^{(18)}$ examined 20 knee specimens to compare the mechanical properties of tibias with PCL avulsion fractures that were fixed with ORIF or arthroscopic sutures; they observed no significant difference in tibial backward shift between the two treatments. It has become apparent that the open posteromedial 
approach has advantages for primary-level hospitals that have difficulties in obtaining access to arthroscopic techniques.

Internal fixation in the current ORIF approaches is often conducted using steel wires, hollow lag screws, absorbable screws and suture anchors. For large fragments, a traditional hollow lag screw may be used. However, in clinical practice, the fracture fragments are usually thin, small or comminuted; for such small fragments, an ideal internal fixation method is still lacking. Conventional screws may not fix the fracture fragments firmly enough and can lead to fragmentation of the bones. Currently, anchors and non-absorbable sutures are often used for ligament reconstruction surgery. Although the ligament can be sutured during surgery, fixation of the fractured bone is not stable and cannot be effectively conducted while applying appropriate pressure to bring the fragments together; therefore, early postoperative functional exercise is not possible. Alternative techniques can provide more stable fixation and potentially better clinical results. For example, Huang et $\mathrm{al}^{(19)}$ used cannulated screws combined with sutures, while Fu et al ${ }^{(4)}$ used the anchor system in combination with hollow lag screws. However, these surgical techniques are cumbersome and the fixation methods complicated, potentially increasing operative time and surgical difficulty. In 2003, Veselko et al ${ }^{(20)}$ reported a simpler method to stably fix fracture fragments using a hollow lag screw combined with a gasket, which could be applied to the management of PCL avulsion fractures of the tibia. Using this approach, fixation can be performed under pressure during surgery. However, the metal gasket disperses the stress of the screw onto the fracture fragments such that the gasket is unable to effectively control the rotational stability of the fracture fragments.

In the present study, a toothed plate was used in combination with a hollow lag screw. We found that this plate was suitable for fracture fragment of all sizes, and allowed for straightforward surgery and short operation time. The toothed plate approach has important advantages. First, the toothed plate expands the fixed area, ${ }^{(21)}$ while the teeth around the plate are embedded into the bone and surface of the ligament to allow the fracture fragments to be fixed using a large torque. Therefore, rotational dislocation was effectively avoided, and the downward and outward stresses caused by the screw tail were dispersed without fear of disintegration of the fracture fragments. Second, the teeth of the plate are able to press part of the ligament fibres into the bone, effectively restoring the tension of the PCL. This results in stable fixation of the fragments and facilitates the healing of bone and ligaments. Finally, the diameter of the toothed plate can be optimally selected for anatomic reduction, based on the sizes of the bone fracture fragments and the extent of fracture.

Based on our experience of the abovementioned surgical procedure, the following points should be noted. Firstly, the displacement of the fracture fragment can lead to the soft tissue becoming embedded in the broken ends of the fractured bone, particularly for old fractures. Therefore, before reduction of the fracture, it is important to completely remove any soft tissue to reveal a fresh bony bed on the tibial eminence, in order to facilitate fracture healing. Secondly, it is important that the anatomic reduction of all fragments is adequate. Reduction of most fracture fragments can be achieved by lifting the femur, conducting an anterior drawer test and pushing the femur forward. If reduction is difficult, traction reduction can be performed using sutures at the end of the PCL. Thirdly, the guide needle needs to be perpendicular to the plate when the hollow lag screw is drilled, in order to produce maximal compressive stress for fragment fixation under pressure when the screw is placed in the plate. Finally, the hollow lag screw should be placed without excessive force, especially for small and thin bone fracture fragments, so as to reduce the risk of ligament necrosis due to excessive pressure.

As the surgical fixation was stable, our patients were able to commence knee function exercises soon after surgery. Nonetheless, the intensity of these exercises was carefully increased in a stepwise manner. We believe that an individualised functional training programme should be developed to facilitate the patients' recovery.

The present study was not without its limitations. First, as this study was a retrospective analysis rather than a randomised controlled trial, a direct comparison with a control group of patients could not be made. Second, due to the small sample size, it is possible that the results cannot be generalised to a wider population. Third, the duration of the follow-up was relatively short, so we were unable to assess long-term clinical efficacy or complications.

In summary, the use of a toothed plate inserted via a posteromedial approach to fix PCL avulsion fractures of the tibia might be a safe, simple and effective procedure suitable for all types of fractures. This approach was associated with satisfactory fixation, minimal complications and good recovery of joint function. Furthermore, this technique could potentially be used at all hospital levels. However, additional larger-scale controlled studies are needed to further evaluate the long-term efficacy of using toothed plates in combination with hollow lag screws for the management of PCL avulsion fractures of the tibia.

\section{REFERENCES}

1. Allen CR, Kaplan LD, Fluhme DJ, Harner CD. Posterior cruciate ligament injuries. Curr Opin Rheumatol 2002; 14:142-9.

2. Sonin AH, Fitzgerald SW, Hoff FL, Friedman H, Bresler ME. MR imaging of the posterior cruciate ligament: normal, abnormal, and associated injury patterns. Radiographics 1995; 15:551-61.

3. Sun HQ, Ren YJ, Zhang YQ. Treatment of posterior cruciate ligament avulsion fracture with pressurized staples. Chi J Orthop Trauma 2011; 13:1189-90

4. Fu YP, Hang CM, Fam HQ. Treatment of posterior cruciate ligament avulsion fracture using anchor system combined with cannulated screw. J Pract Orthop 2011; 17:73-4.

5. Lysholm J, Gillquist J. Evaluation of knee ligament surgery results with special emphasis on use of a scoring scale. Am J Sports Med 1982; 10:150-4.

6. Tajima G, Nozaki M, Iriuchishima T, et al. Morphology of the tibial insertion of the posterior cruciate ligament. J Bone Joint Surg Am 2009; 91:859-66.

7. Janousek AT, Jones DG, Clatworthy M, Higgins LD, Fu FH. Posterior cruciate ligament injuries of the knee joint. Sports Med 1999; 28:429-41.

8. Schulz MS, Russe K, Weiler A, Eichhorn HJ, Strobel MJ. Epidemiology of posterior cruciate ligament injuries. Arch Orthop Trauma Surg 2003; 123:186-91

9. Ogawa H, Sumi H, Shimizu K. Posterior cruciate ligament mediated avulsion fracture of the lateral tibial condyle: a case report. J Orthop Surg Res 2010; 5:67. 
10. Strobel MJ, Weiler A, Schulz MS, Russe K, Eichhorn HJ. Arthroscopic evaluation of articular cartilage lesions in posterior-cruciate-ligamentdeficient knees. Arthroscopy 2003; 19:262-8.

11. Wang J, Ao YF. [The effect of rupture and reconstruction of posterior cruciate ligament on the degeneration of articular cartilage in rabbit knee] Zhonghua Wai Ke Za Zhi 2005; 43:1598-601. Chinese.

12. Ugutmen E, Sener N, Eren A, Beksac B, Altintas F. Avulsion fracture of the posterior cruciate ligament at the tibial insertion in a child: a case report. Knee Surg Sports Traumatol Arthrosc 2006; 14:340-2.

13. Anderson AF, Anderson CN. Posterior cruciate and posterolateral ligament reconstruction in an adolescent with open physes. A case report. J Bone Joint Surg Am 2007; 89:1598-604.

14. Zhao JZ, He YH, Wang JH. Treatment of acute posterior cruciate ligament avulsion fracture via arthroscopically assisted double posteromedial approach. Chi J Orthop 2005; 25:16-20.

15. Lu HX, Huangpu XQ, Zhang J. Treatment of acute posterior cruciate ligament avulsion fracture with arthroscopically assisted cannulated screw fixation. J Pract Orthop 2012; 18:797-9.

16. Horas U, Meissner SA, Heiss C, Schnettler R. Arthroscopic fixation of posterior cruciate ligament avulsion fractures: a new minimally invasive technique. Knee Surg Sports Traumatol Arthrosc 2010; 18:781-3.

17. Chen W, Tang D, Kang L, Ding Z, Sha M, Hong J. Effects of microendoscopyassisted reduction and screw fixation through a single mini-incision on posterior cruciate ligament tibial avulsion fracture. Arch Orthop Trauma Surg 2012; 132:429-35.

18. Sasaki SU, da Mota e Albuquerque RF, Amatuzzi MM, Pereira CA. Open screw fixation versus arthroscopic suture fixation of tibial posterior cruciate ligament avulsion injuries: a mechanical comparison. Arthroscopy 2007; 23:1226-30

19. Huang CM, Fan HQ, Wang JX. Short term outcomes of arthroscopically assisted treatment for posterior cruciate ligament avulsion fracture. Orthop J China 2009; 17:272-5

20. Veselko M, Saciri V. Posterior approach for arthroscopic reduction and antegrade fixation of avulsion fracture of the posterior cruciate ligament from the tibia with cannulated screw and washer. Arthroscopy 2003; 19:916-21.

21. Rovinsky D, Haskell A, Liu Q, Paiement GD, Robinovitch S. Evaluation of a new method of small fragment fixation in a medial malleolus fracture model. J Orthop Trauma 2000; 14:420-5. 